\title{
An unusual infection of visceral compartment of the neck following retropharyngeal space infection.
}

\author{
Dr. Vijay Bidkar ${ }^{1}$, Dr. Ashok Naik ${ }^{2}$, Dr. Raghunath Shanbag ${ }^{3}$, Dr. Roshan \\ Jalisattigi ${ }^{4}$, Dr. Rashmi.S. ${ }^{5}$ \\ ${ }^{1}$ Assistant Professor. Department of ENT, SDM College of Medical Sciences \& Hospital. Dharwad, Karnataka, \\ India. \\ ${ }^{2}$ Professor. Department of ENT, SDM College of Medical Sciences \& Hospital. Dharwad, Karnataka, India. \\ ${ }^{3}$ Professor. Department of ENT, SDM College of Medical Sciences \& Hospital. Dharwad, Karnataka, India. \\ ${ }^{4}$ Assistant Professor. Department of ENT, SDM College of Medical Sciences \& Hospital. Dharwad, Karnataka, \\ India. \\ ${ }^{5}$ Senior Resident .Department of ENT, SDM College of Medical Sciences \& Hospital. Dharwad, Karnataka, \\ India.
}

\begin{abstract}
A fifty year old male, diagnosed to have an acute retropharyngeal space infection reported two days after his discharge from the hospital with breathing difficulty and rapidly progressive diffuse swelling of the neck. Immediate clinical evaluation failed to localize the exact cause but computed tomography revealed large anterior visceral compartment abscess with recurring retropharyngeal space infection. In this case the involvement of visceral compartment of the neck was a unique presentation.

Retropharyngeal space infections are known for their spread to other spaces. However, involvement of visceral compartment is rare. Proximity to visceral vascular space and possibility of spread of infection to superior mediastinum makes visceral space infections a life threatening condition.

Deep neck space infections should be observed for extensions beyond the primary site. The role of imaging is indispensable while monitoring these infections. Prompt initiation of definitive treatment can prevent catastrophic complications.
\end{abstract}

Keywords - Visceral compartment of neck, retropharyngeal space infection, computed tomography scan.

\section{Introduction}

The cervical fascia compartmentalizes the neck into potential spaces. Infection from one space can spread to another along the fascial planes ${ }^{1}$. The visceral compartment is a nonpotential infrahyoid deep neck space that includes visceral organs of the neck thyroid, trachea and esophagus enclosed by visceral layer of deep cervical fascia.

Retropharyngeal space is one of the major deep neck space related to upper aero digestive passage. This space is divided by midline fibrous raphe. Infection with sufficient oncotic pressure may either cross the midline ${ }^{2}$ or infect other spaces along fascial planes. In the infrahyoid region visceral compartment lies anterior to the retropharyngeal space. The middle layer of deep cervical fascia projects anteriorly, where it is known as "anterior visceral fascia." Inferiorly, anterior visceral fascia fuses with the parietal pericardium and adventitia of great vessels. Therefore infections of retropharyngeal space can track around the visceral space and extend down in to the superior mediastinum. ${ }^{3}$

Acute retropharyngeal space infection in a middle age man without any pre existing co morbidities is relatively rare, unless as a result of trauma to pharynx by ingestion of foreign body or during instrumentation of upper aero-digestive tract. ${ }^{4}$ However, it is more common among children due to abundance of lymph nodes in this space. These lymph nodes usually disappear by the age of 4 to 5years., ${ }^{4,5}$

We describe a rare case of visceral compartment infection following an acute retropharyngeal space infection in a fifty year old male after traumatic injury to the upper aero digestive tract. Initially the patient recovered from retropharyngeal space infection after a course of intravenous antibiotics and was discharged from the hospital satisfactorily. Two days later he developed cervical pain, redness and a diffuse swelling over the neck. Computed tomography revealed a large swelling in the anterior visceral compartment. Extension of the infection from retropharyngeal space was unanticipated after an early recovery with conservative management.

\section{Case report}

A fifty year man presented with neck pain, dysphagia and fever two days following accidental ingestion of a large piece of meat. On examination his ear, nose and throat were unremarkable. Full blood counts showed neutrophilia with leukocytosis. Plain radiograph of soft tissue neck lateral view (Figure 1) 
showed widening of retropharyngeal space at C2 vertebral level. Provisionally it was diagnosed as, post traumatic acute retropharyngeal space infection. Intravenous antibiotics, parentral fluids and anti inflammatory drugs were administered for two days. Because of persistence of symptoms we undertook the patient for rigid direct laryngoscopy under general anesthesia. A small tear over mucosa of posterior pharyngeal wall was seen. Intravenous antibiotics and nasogasrtic feeds were continued for seven more days allowing the ulcer to heal. Patient was discharged with oral feeding establishment after complete recovery.

Two days later, patient came back to casualty. He was having diffuse swelling, redness and raised local temperature of overlying skin of the neck. Computed tomography (CT) scan of the neck revealed, a fairly well defined large multiloculated thick peripheral wall enhancing abscess predominantly in the infrahyoid neck space on the right side. The largest dimension of the abscess on CT scan was $115 \times 55 \times 50 \mathrm{~mm}$. Widening of the retropharyngeal space along with appearance of abscess in infrahyoid region was seen on axial section of CT scan of the neck (Figure 2).Also seen was a mild posterolateral displacement of the superior aspect of the right lobe of thyroid by an enlarging abscess cavity (Figure 3) and an inferior extension into the superior mediastinum (Figure 4). A diagnosis of a concurrent infection of visceral compartment with retropharyngeal space infection was confirmed on radiological studies as described above.

The patient was then considered for incision and drainage of the visceral compartment abscess. The abscess was drained under general anesthesia. A horizontal skin crease incision was taken two fingers breadth above the sternoclavicular joint on the right side. Soft tissue dissection between medial border of the sternocleidomastoid and strap muscles was carried out. The thick wall of abscess cavity was identified and was opened. Approximately 25 to $30 \mathrm{cc}$ of thick purulent material was drained and sent for microbiological evaluation. Daily dressing of the abscess cavity was done.

\section{Discussion:}

Spread of infection in the deep neck spaces is mainly attributed to anatomical continuity and close proximity of these spaces to each other. Retropharyngeal abscess and its spread to other spaces elsewhere in head, neck and chest is well documented in the literature. However, spread into visceral compartment is poorly discussed in the literature.

Hollinshed classified infrahyoid neck spaces as I. Visceral compartment II. Visceral space and III. Other spaces ${ }^{5}$.The visceral compartment contains thyroid gland, trachea and esophagus along with loose aereolar tissue (Figure 5). At the level of entry of inferior thyroid artery in to the thyroid gland, it is further subdivided by a dense connective tissue attaching esophagus laterally to the carotid sheath. The anterior part of the compartment containing trachea is known as previsceral or pretracheal space. The posterior part lying behind the pharynx and esophagus is known as retrovisceral, retropharyngeal, retroesophageal or post visceral space. Pre-tracheal space is superiorly limited by attachment of strap muscles and their fascia to the thyroid cartilage and hyoid bone, while inferiorly it is continuous with superior mediastinum and extends up to the upper border of arch of aorta where it is limited by dense adhesions between fibrous pericardium and posterior surface of sternum. Laterally it is blind at the root of the neck because of dense adhesions between alar and visceral fasciae. This space can get infected by trauma to the anterior part of the esophagus and also sometimes by infections of the contents of retrovisceral space. Surgically this space can be opened by an incision anterior to sternocleidomastoid muscle. Grodinsky and Holyoke, described the retrovisceral space ${ }^{6,7}$ which extends from the base of skull superiorly to the superior mediastinum inferiorly. Pearse observed retrovisceral space as an important focus and a route for the spread of infections originating in the head and upper portion of the neck to the superior mediastinum ${ }^{8}$ and has reported it to be as high as $71 \%$. Retrovisceral space may be infected by posterior perforation of esophagus or infection of deep cervical lymph nodes. Iglauer described an approach to this space by an incision posterior to the Sternocleidomastoid ${ }^{\mathbf{9}}$. The visceral space is an imaginary space which exists between visceral fascia and the organs themselves (trachea or esophagus).Esophagus is enclosed in a connective tissue sheath which is continuous superiorly with the buccopharyngeal fascia over the posterior surface of pharynx. Extension of the same facial sheath encloses the trachea and thyroid gland individually. Grodinsky and Holyoke labeled this as visceral fascia, ${ }^{\mathbf{6}, 7}$ the visceral fascia is firmly attached to the underlying structures. Therefore existence of visceral space is a matter of debate. Any infection lying deeper to this fascia tends to reach the visceral compartment rather spreading cranio caudally along the space. Other space includes the cavity within the carotid sheath. The carotid sheath along with the visceral space is grouped under visceral vascular space by Coller and Yglesias who pointed out that infection from the visceral space can readily spread to this potential cavity within the carotid sheath, later also being a pathway for the spread of infections from upper to the lower part of the neck and into the mediastinum ${ }^{10,11}$. According to Pearse, $21 \%$ of mediastinal suppurations originating in the neck spread along this pathway.

Acute onset retropharyngeal abscess in an otherwise healthy individual occurs most commonly following local trauma, foreign bodies, or as a complication of dental infections. Retropharyngeal abscess is more common in males than in females, with reported male preponderance of $53-55 \%$. The principal symptoms 
in adults are throat pain, fever, difficulty in swallowing, odynophagia and neck pain. Patients with retropharyngeal abscesses may have signs of airway obstruction, but often they do not. The most common physical presentation is posterior pharyngeal oedema $37 \%$, nuchal rigidity, cervical adenopathy, drooling, and stridor. ${ }^{12}$ A study by Ridder et al in 234 adults with deep neck space infections, observed the mortality rate of $2.6 \%{ }^{13}$ The cause of death was primarily sepsis with multiorgan failure. In our case patient gives history of ingestion of meat before he developed retropharyngeal abscess. Direct laryngoscopy confirmed trauma to posterior pharyngeal wall. Diagnosis of a retropharyngeal abscess was confirmed by a plain lateral view radiograph of soft tissue of the neck. In absence of obvious swelling over the neck, spread of infection in visceral compartment remained concealed.

Most of these abscesses in adults are polymicrobial with predominant organisms being Staphylococcus aureus and group A beta hemolytic Streptococcus. Other organisms isolated are anaerobic organisms (species of Bacteroides and Veillonella), or Gram-negative organisms (Haemophilus parainfluenzae and Bartonella henselae).

When establishing a diagnosis in prototypical retropharyngeal abscess, a plain lateral view radiograph of soft tissue of the neck is very helpful, but CT scan has enormous value as a diagnostic tool that can detect an abscess and help to establish treatment regimen. ${ }^{14}$

Most authors recommend a combined treatment of antibiotics with surgical drainage for management of non tuberculous retropharyngeal abscess. ${ }^{15}$ Intraoral drainage is preferred if the abscess is confined to the level above hyoid bone. If it extends below this level, it should be drained externally. ${ }^{16}$ Spread of infection to the visceral compartment of the neck needs an external incision apart from intraoral drainage and antibiotics as in our case.

\section{Conclusion}

Concurrent involvement of the visceral compartment of the neck following infection of retropharyngeal space is relatively rare.

High index of suspicion is required in diagnosing unusual spread of infection from retropharyngeal space. Delayed presentation in our patient was due to ability of neck to accommodate large abscess cavity without obvious symptoms. Many physicians are not familiar with such an unusual spread of infection. Hence all the patients of deep neck space infections should be kept under observation for early diagnosis of concurrent or secondary spread of infection.

CT scan of the neck is more informative than plain radiograph for radiological evaluation of these deep neck spaces.

A retropharyngeal abscess needs antibiotics and surgical drainage. However, a concurrent visceral compartment infection warrants a separate external incision and drainage as described.

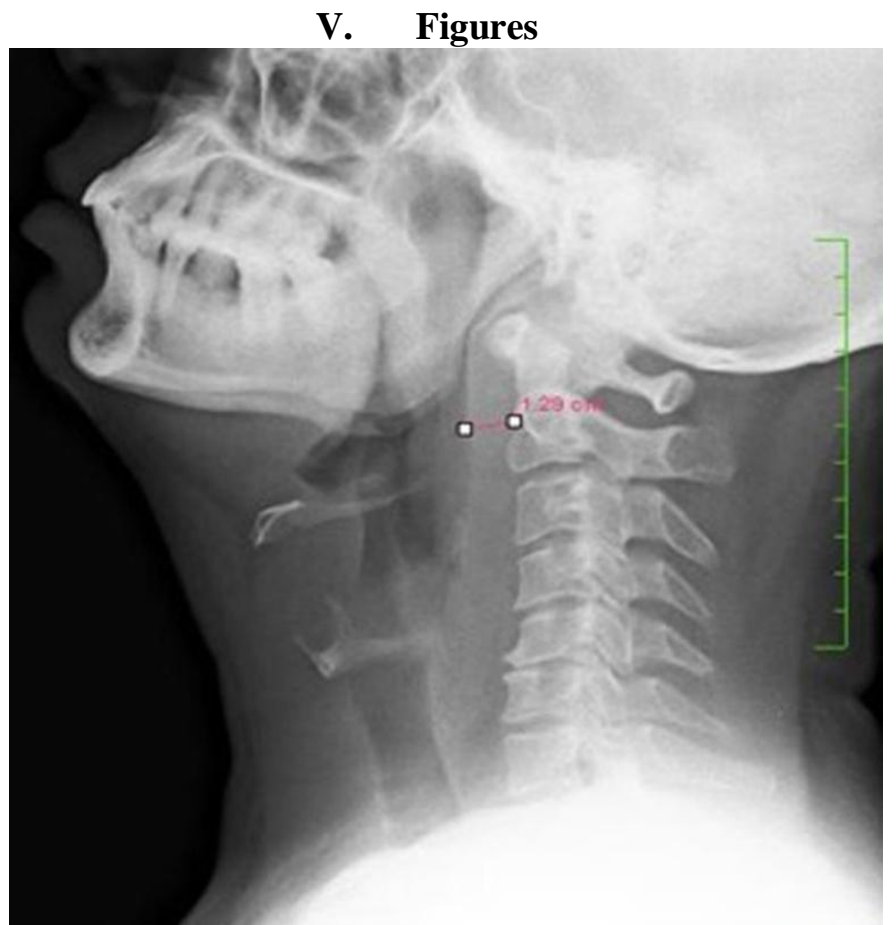

Figure 1: Plain radiograph lateral view of neck showing widened retropharyngeal space at $\mathrm{C} 2$ vertebral level. 


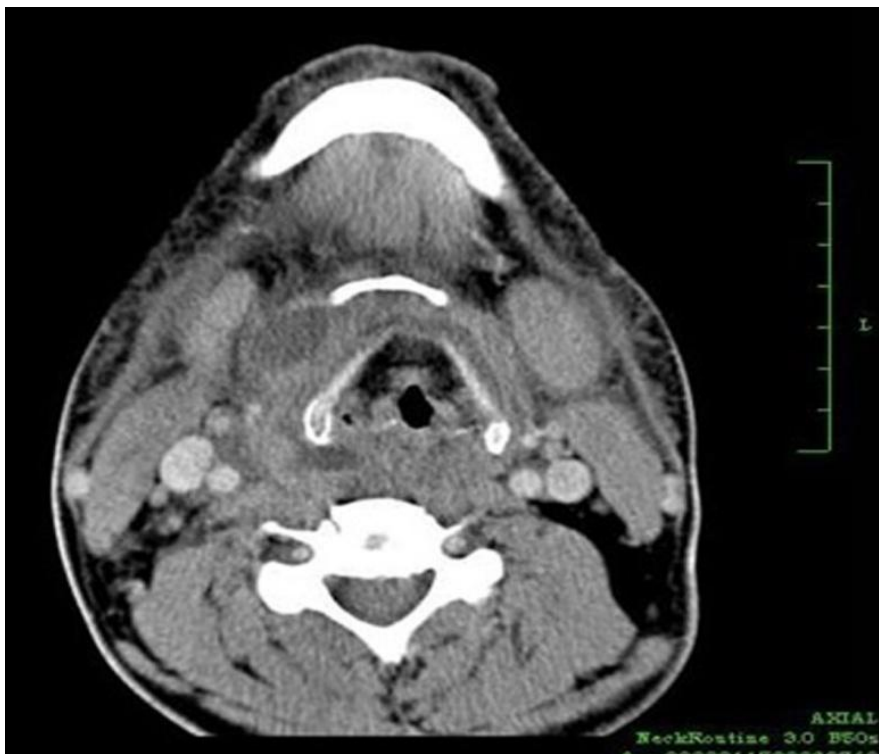

Figure 2: Contrast $\mathrm{CT}$ axial section of neck showing abscess in right infrahyoid space with collection in retropharyngeal space.

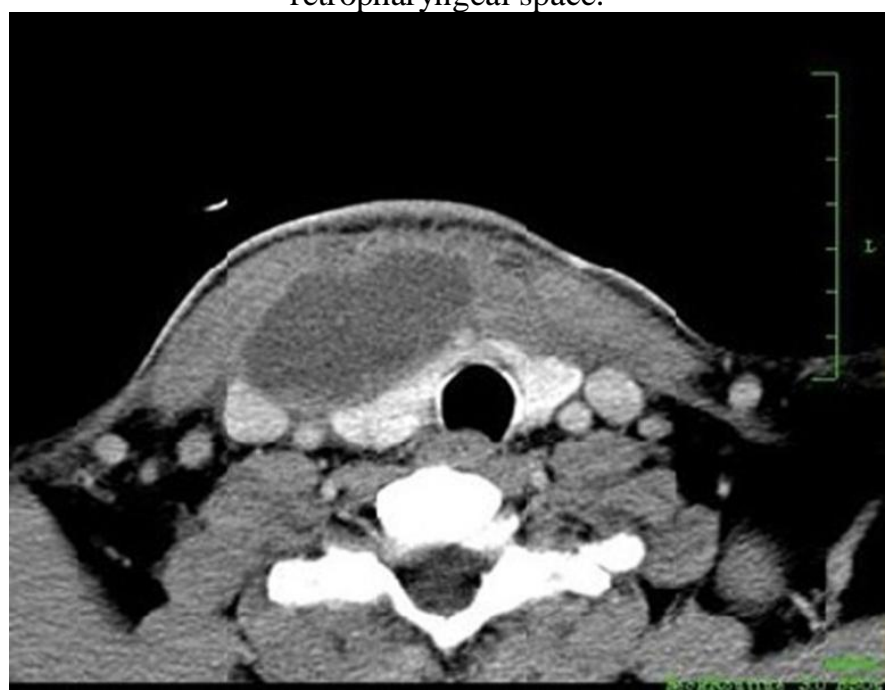

Figure 3: Contrast CT axial section of neck showing posterolateral displacement of right lobe of thyroid gland.

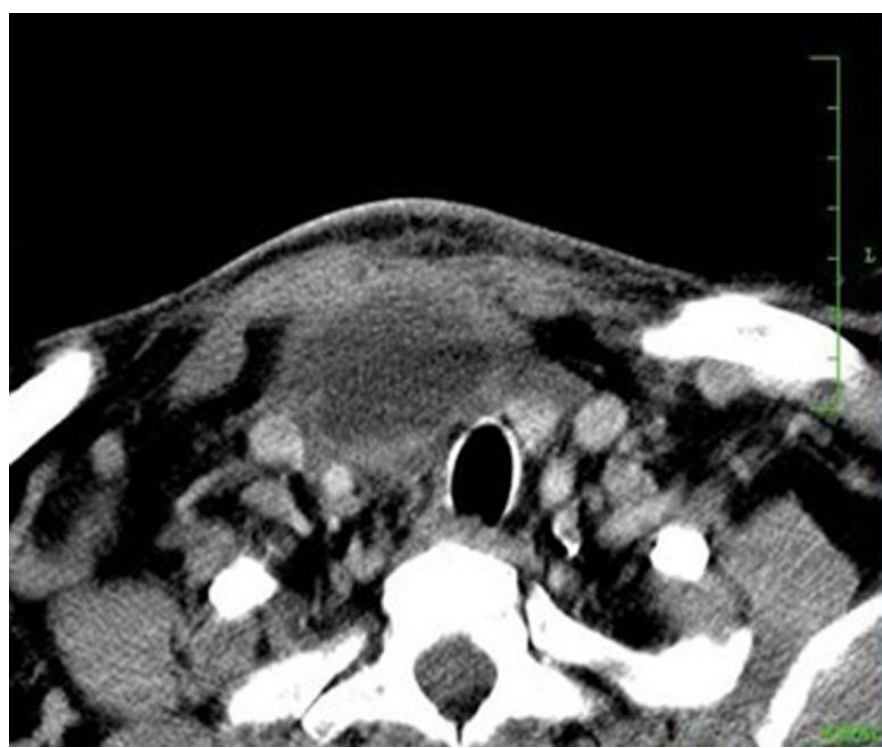

Figure 4: Contrast CT axial section of neck showing extension of abscess cavity in superior mediastinum. 


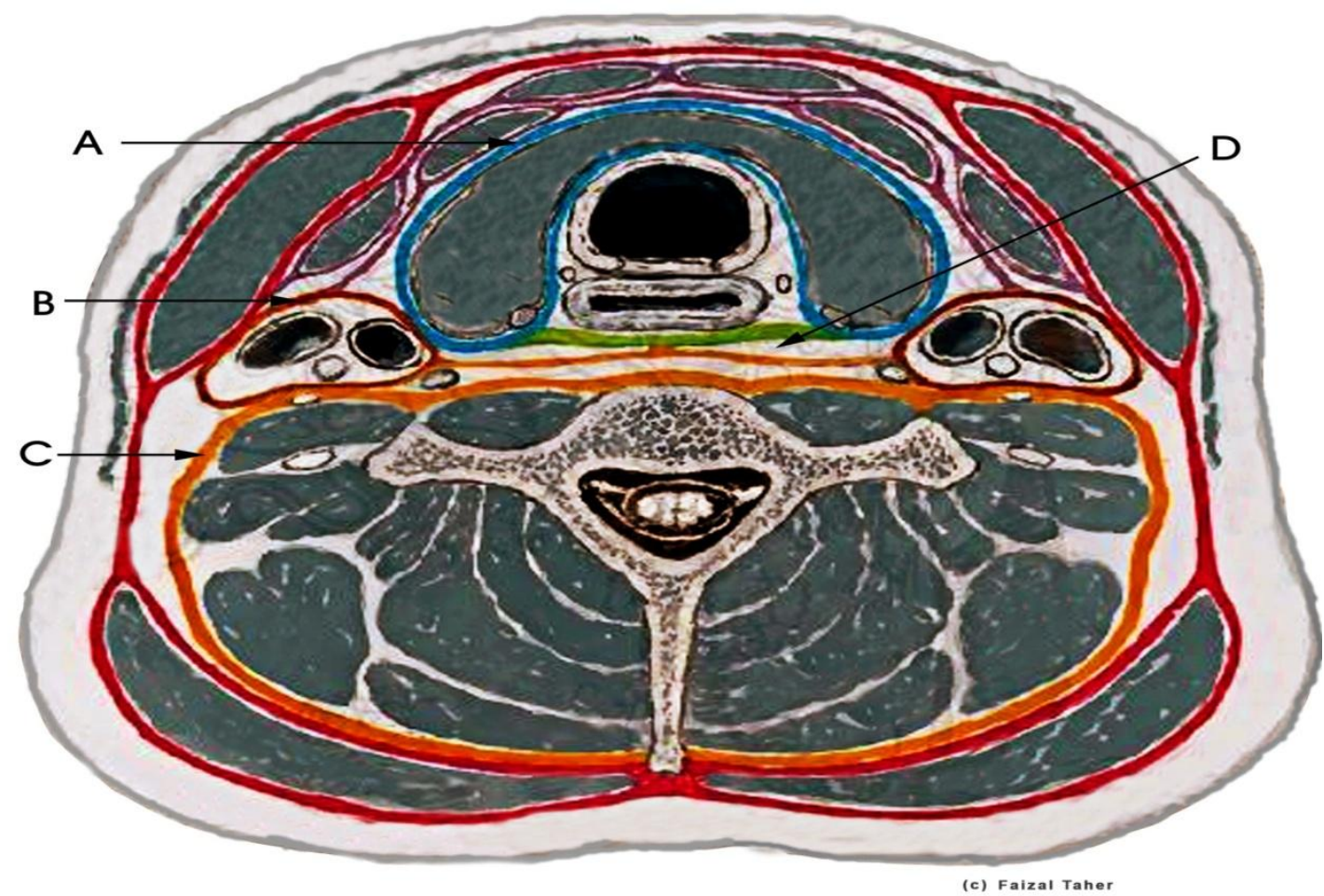

Figure 5: Diagrammatic presentation of the neck, showing superficial and deep layers of cervical fascia enclosing various spaces and their contents. A-Visceral compartment containing thyroid gland, trachea and esophagus, B- Carotid Space, C- Prevertebral space, D- Retropharyngeal space.

\section{References}

[1] Gidley PW, Ghorayeb BY, Stiernberg CM. Contemporary management of deep neck space infections. Otolaryngol Head Neck Surg. 1997 Jan; 116(1):16-22. [Pub med]

[2] Eugene N Myers, Operative otolaryngology Head and Neck Surgery, Deep neck space infection, John T Jhonson. vol.1, chapter 77:p672-673

[3] Steven CR, Anthony WC. Life threatening infections of peripharyngeal and deep fascial spaces of the Head and Neck. Vol. 21, Infectious disease clinics of North America (2007): pp. 569-570.

[4] Arora S, Sharma JK, Pippal SK, Yadav A, Najmi M, Singhal D. Retropharyngeal abscess following a gunshot injury. Brazilian Journal of Otorhinolaryngology. 2009; 75(6):p. 909.

[5] Marques PM, Spratley JE, Leal LM, Cardoso E, Santos M. Parapharyngeal abscess in children: five year retrospective study. Brazilian Journal of Otorhinolaryngology. 2009; 75(6):826-830.

[6] Grodinsky, M and Holyoke, E. A. (1938): The fasciae and fascial spaces of head, neck and adjacent regions. American Journal of Anatomy 63: p. 367-93.

[7] Grodinsky M, (1939): Retropharyngeal and lateral pharyngeal abscesses: An anatomic and clinical study. Annzeles Surgery 110: p. 177.

[8] Pearse, H. E. (1938): Mediastinitis following cervical suppuration. Annzeles Surgery 108: p. 588.

[9] Iglauer, S. (1935): Surgical approaches to deep suppuration in the neck and posterior mediastinum. Archives Otolaryngology 21: p. 707.

[10] Coller, F. A. and Yglesias, L. (1935): Infections of the lip and face. Surgery, Gynecology and Obstetrics, 60: p. 277.

[11] Coller, F. A. and Yglesias, L. (1937): The relation of the spread of infection to fascial planes in the neck and thorax. Surgery 1: p. 323

[12] Pollard BA, El-Beheiry H. Pott's disease with unstable cervical spine, retropharyngeal cold abscess and progressive airway obstruction. Canadian Journal of Anesthesia. 1999; 46(8):772-775.

[13] Ridder GJ, Technau-Ihling K, Sander A, Boedeker CC. Spectrum and management of deep neck space infections: an 8-year experience of 234 cases. Otolaryngology_Head and Neck Surgery.2005; 133(5):709-714.

[14] Oh JH, Kim Y, Kim CH. Parapharyngeal abscess: comprehensive management protocol. ORL J Otorhinolaryngol Relat Spec. 2007; 69:37-42.

[15] Wang LF, Kuo WR, Tsai SM, Huang KJ. Characterizations of life-threatening deep cervical space infections: a review of one hundred ninety-six cases. American Journal of Otolaryngology.2003; 24(2):111-117.

[16] Bluestone CD, Rosenfield RM. Surgical atlas of pediatric otolaryngology. In: Decker BC, editor. London: Inc. Hamilton; 2002. pp. 482-6. 